\title{
Tratamiento con tofacitinib en adolescentes con alopecia areata
}

\author{
Adalid Y. Morales-Miranda ${ }^{1 *}$, Guadalupe M. Bueno-Arias ${ }^{2}$, Óscar G. Aguirre-Félix ${ }^{1}$ y Rocío Tovar-Franco ${ }^{3}$ \\ ${ }^{1}$ Unidad de Especialidades Médicas; ${ }^{2}$ Escuela Militar de Graduados de Sanidad; ${ }^{3}$ Servicio de Dermatología, Unidad de Especialidades Médicas. \\ Universidad del Ejército y Fuerza Aérea, Secretaría de la Defensa Nacional, Ciudad de México, México
}

\begin{abstract}
Resumen
Introducción: Actualmente no existe un tratamiento estandarizado para la alopecia areata (AA) grave. Se han reportado numerosos casos exitosos del uso de tofacitinib; sin embargo, no existen publicaciones en México. En este trabajo se reportan cuatro casos de pacientes mexicanos con AA grave tratados con tofacitinib oral. Métodos: Serie de casos de adolescentes con alopecia grave tratados con tofacitinib oral. Para determinar la respuesta al tratamiento se utilizó la Escala de gravedad de alopecia (Severity of alopecia tool). Resultados: Se incluyeron cuatro pacientes de entre 13 y 19 años con AA. En todos los casos se observó crecimiento de cabello y disminución de la gravedad de la alopecia después del tratamiento con tofacitinib. En dos pacientes se observó una respuesta intermedia (del 51 al 90\%), y en los otros, moderada (del 6 al 50\%), sin efectos adversos serios. Las limitaciones del estudio fueron el tamaño reducido de la muestra y la naturaleza retrospectiva de la recolección de los datos. Conclusiones: El tofacitinib demostró ser una buena alternativa de tratamiento para la $A A$, total y universal, refractarias a otras terapias.
\end{abstract}

Palabras clave: Alopecia areata. Tofacitinib. Janus kinases (JAK).

\section{Tofacitinib as a treatment of alopecia areata in adolescents}

\begin{abstract}
Background: Currently, no standardized treatment for severe alopecia areata (AA) exists. Numerous successful cases of the use of tofacitinib have been reported in the world literature, but not in Mexico. Four Mexican adolescents with severe $A A$ treated with oral tofacitinib are reported in the present work. Methods: Series of cases of adolescents with severe AA treated with oral tofacitinib. The severity of alopecia tool was used to determine the response to treatment. Results: Four patients from 13 to 19 years old, were included. In all cases, hair growth was observed, and the alopecia severity decreased after the treatment with tofacitinib. In two patients, an intermediate response (from 51 to 90\%) was observed; in the other, a moderate response (from 6 to 50\%) was observed, without serious adverse effects. The limitations of the study were the small sample size and the retrospective nature of data collection. Conclusions: Tofacitinib showed to be a good treatment alternative for $A A$, total and universal, refractory to other therapies.
\end{abstract}

Key words: Alopecia areata. Tofacitinib. Janus kinases (JAK).

Correspondencia:

*Adalid Yakelin Morales-Miranda

E-mail: morayami05@hotmail.com
Fecha de recepción: 16-01-2019

Fecha de aceptación: 29-04-2019

DOI: 10.24875/BMHIM.19000005
Disponible en internet: 05-07-2019 Bol Med Hosp Infant Mex. 2019;76:182-187 www.bmhim.com 


\section{Introducción}

La alopecia areata (AA) es una afección autoinmunitaria órgano-específica caracterizada por placas sin pelo, de inicio súbito, localizadas con mayor frecuencia en piel cabelluda, aunque pueden afectar cualquier área pilosa ${ }^{1}$. El pronóstico de este padecimiento es variable; se ha descrito que hasta el $50 \%$ de los casos de las placas sin pelo pueden repoblarse de manera espontánea en el lapso de un año. Sin embargo, del 10 al $15 \%$ de los pacientes pueden evolucionar a las formas graves de la enfermedad (alopecia total o alopecia universal). Estas variedades tienden a la cronicidad y presentan una tasa de repoblación menor al $15 \%$ $\%^{2,3}$.

A la fecha, debido a la falta de ensayos clínicos, no existe un tratamiento aprobado ni estandarizado para la AA grave. La principal dificultad para la realización de estos ensayos reside en el alto porcentaje de recuperación espontánea, baja frecuencia y mal pronóstico en los casos leves ${ }^{4}$. Las guías de tratamiento disponibles en la actualidad para casos con extensión mayor al $50 \%$ de la superficie de la piel cabelluda afectada recomiendan la administración de esteroides sistémicos, ciclosporina, metotrexato, y la inmunoterapia de contacto con ácido escuárico o difenilciclopropenona ${ }^{5}$, la cual no se encuentra disponible en México. Otras terapias reportadas en la literatura con menor evidencia de eficacia son el plasma rico en plaquetas, la aromaterapia, la meditación mindfulness, entre otras ${ }^{5,6}$. Si bien se observa repoblación parcial de los parches alopécicos en la mayoría de las ocasiones, la regla son las recaídas al suspender el tratamiento, por lo que no se ha demostrado cambio en la evolución de la enfermedad ${ }^{7}$.

Estudios recientes han demostrado que los linfocitos $\mathrm{T}$ citotóxicos producen interferón gamma (IFN- $\gamma$ ) en respuesta a la activación por la interleucina 2 y 15 (IL-2 e IL-15), interviniendo de manera determinante en la patogenia de la AA. Estos hallazgos y numerosas investigaciones en modelos murinos han demostrado que los inhibidores de la vía JAK/STAT (ruloxitinib y tofacitinib) pueden tener efectos terapéuticos benéficos en distintas enfermedades inflamatorias ${ }^{8,9}$.

El tofacitinib es una molécula pequeña Janus cinasa 1/3 (JAK 1/3), aprobada por la Food and Drug Administration a finales del año 2012 como tratamiento para la artritis reumatoide moderada a grave. Los primeros reportes de su uso en AA se publicaron en el año $2014^{10,11}$. En México, al presente día, no existen reportes al respecto.

\section{Métodos}

Se obtuvieron y revisaron los expedientes clínicos de pacientes de 13 a 19 años con AA universal (AU) y total (AT), con afectación mayor al $50 \%$ de la superficie total de la cabeza, atendidos por el Servicio de Dermatología de tercer nivel en la Ciudad de México, entre enero y diciembre del 2018. Los pacientes recibieron tofacitinib (5 mg) cada 12 horas, vía oral, por un periodo mínimo de 6 meses. Los pacientes no habían recibido ningún tratamiento para la $\mathrm{AA}$ en los tres últimos meses. Se realizaron estudios de laboratorio: biometría hemática y perfil hepático, así como PPD (derivado proteico purificado) y perfil viral para hepatitis $\mathrm{B}$ y $\mathrm{C}$.

Se registró la edad, sexo, tiempo de evolución con la alopecia, tiempo de evolución del episodio actual, edad al inicio de la alopecia, antecedente familiar de AA, comorbilidades, tratamientos previos y gravedad de la alopecia medida según la Escala de gravedad de alopecia (SALT, por sus siglas en inglés, severity of alopecia tool) al inicio, a los 3 y a los 6 meses de tratamiento. La respuesta al tratamiento se evaluó con la reducción de la gravedad determinada por la escala SALT (donde 100 es igual a ausencia total de cabello y 0 repoblación total), y se clasificó en respuesta completa (>90\% de mejoría del SALT), respuesta intermedia (del 51 al $90 \%$ ), respuesta moderada (del 6 al 50\%) y ausencia de respuesta $(<5 \%)$, según lo propuesto por Liu, et al. ${ }^{12}$. Así mismo, se determinaron los efectos adversos y se evaluó la satisfacción del paciente con el tratamiento, considerando una escala del 0 al 3 , donde $0=$ muy insatisfecho, $1=$ insatisfecho, 2 = satisfecho y $3=$ muy satisfecho.

\section{Resultados}

Se encontraron cuatro pacientes, dos hombres y dos mujeres, con un rango de edad de 13 a 19 años (mediana de 15 años). Dos pacientes tenían AU, uno AT y uno AA en placas con afección mayor al $50 \%$. El tiempo de evolución del último episodio fue de 2 a 5 años (mediana de 3 años). Con respecto a las comorbilidades, una paciente presentó tiroiditis autoinmune y anemia por deficiencia de hierro; dos pacientes presentaron deficiencia de vitamina $D$, tratada antes del inicio del tofacitinib. Los tratamientos para la AA utilizados previamente por los pacientes fueron minoxidil en loción (cuatro pacientes), propionato de clobetasol (dos pacientes), prednisona oral (cuatro pacientes), metotrexato (tres pacientes), fototerapia (un paciente), ciclosporina (un paciente) y simvastatina/ezetimiba (dos pacientes). Estos tratamientos fueron utilizados por periodos de 3 


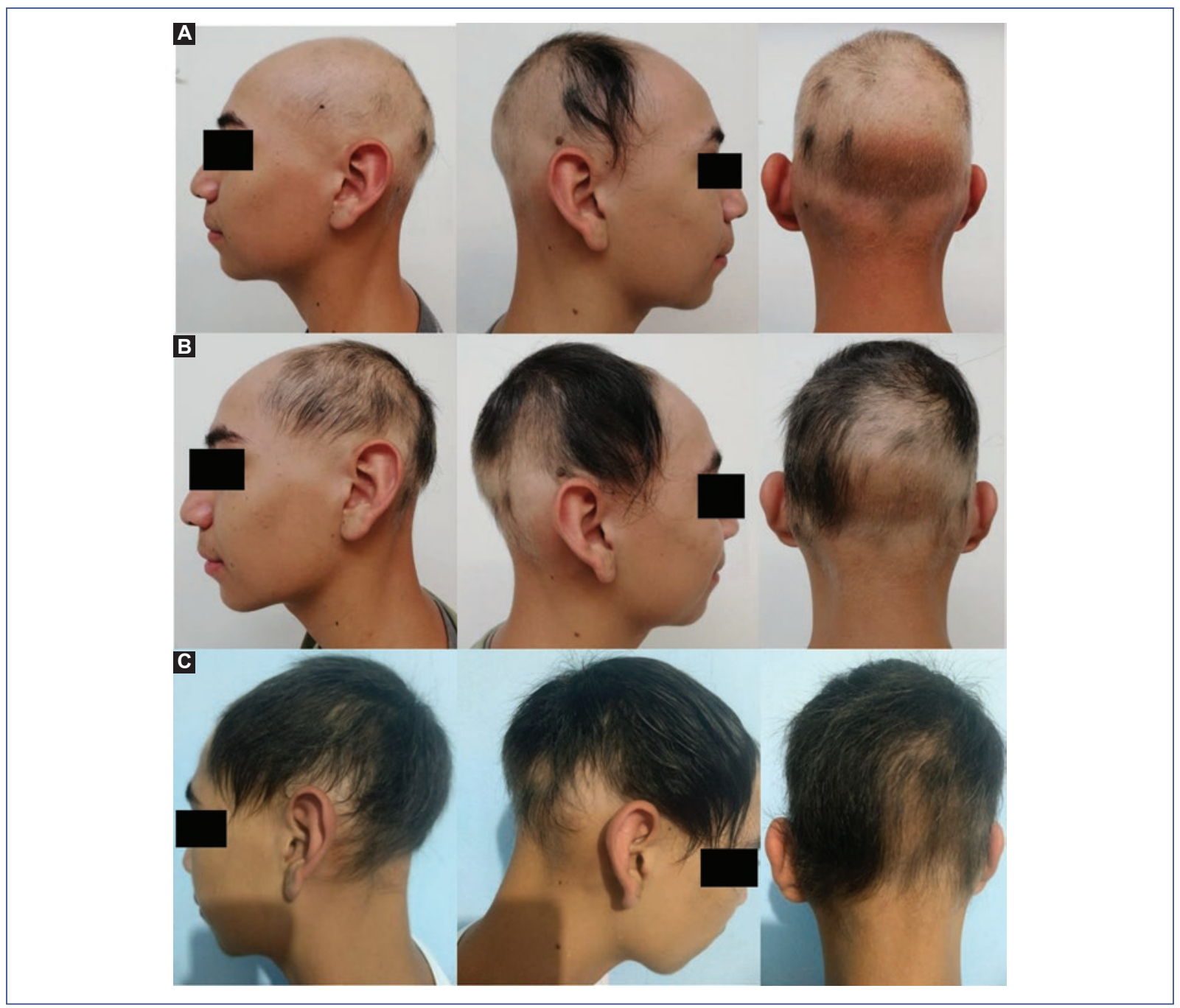

Figura 1. Adolescente de 14 años de edad con alopecia areata: antes del inicio del tratamiento con tofacitinib (A), evolución a los 3 meses (B) y a los 6 meses de tratamiento (C).

a 6 meses, con repoblación menor al $5 \%$ de las placas sin pelo. Las características demográficas, el tiempo de evolución, edad de inicio y tratamientos previos de cada paciente se describen en la tabla 1. El perfil viral para hepatitis $B$ y $C$ fue negativo en los cuatro casos.

Después de 6 meses de tratamiento, los cuatro pacientes mostraron crecimiento de cabello y disminución en la gravedad de la alopecia determinada mediante la escala SALT. Dos pacientes mostraron respuesta intermedia: la gravedad de la AA disminuyó del 97 inicial al $16 \%$ y del 70 inicial al 19\%, respectivamente. Dos pacientes tuvieron respuesta moderada, en quienes la gravedad inicial disminuyó del 99 al $70 \%$ y del 96 al $60 \%$, respectivamente (Fig. 1). Con respecto a la satisfacción de los pacientes con los resultados obtenidos, el $50 \%$ se manifestaron satisfechos, y el otro $50 \%$, insatisfechos. Ningún paciente presentó efectos adversos secundarios graves ni cambios en los estudios de laboratorio. Un paciente reportó infección de las vías respiratorias altas (faringoamigdalitis), por lo que se le administró un antibiótico oral, sin ameritar la suspensión del tratamiento. Actualmente, los pacientes continúan con el tratamiento con tofacitinib ( $5 \mathrm{mg}$ al día).

Los cambios en la escala de SALT en cada paciente después de 3 y 6 meses de tratamiento, la satisfacción con el tratamiento, así como la presencia de efectos adversos se muestran en la tabla 2.

\section{Discusión}

Desde 2014, existen numerosas publicaciones de adultos con AA tratados con tofacitinib oral y tópico, en las que se observa repoblación del 56 al $70 \%$ de las placas sin pelo, con mejoría de la gravedad del 77 al 
Tabla 1. Características demográficas de la población estudiada

\begin{tabular}{|c|c|c|c|c|c|c|c|c|}
\hline Paciente & $\begin{array}{l}\text { Edad } \\
\text { (años) }\end{array}$ & Sexo & $\begin{array}{l}\text { Edad de inicio } \\
\text { de la AA (años) }\end{array}$ & $\begin{array}{l}\text { Antecedente } \\
\text { familiar de AA }\end{array}$ & Comorbilidades & $\begin{array}{l}\text { Evolución del } \\
\text { episodio actual }\end{array}$ & $\begin{array}{l}\text { Variedad } \\
\text { clínica }\end{array}$ & $\begin{array}{l}\text { Tratamientos } \\
\text { previos }\end{array}$ \\
\hline 1 & 13 & $\mathrm{~F}$ & 3 & No & $\begin{array}{l}\text { Tiroiditis } \\
\text { autoinmune } \\
\text { Anemia } \\
\text { microcítica } \\
\text { hipocrómica } \\
\text { Deficiencia de } \\
\text { vitamina D }\end{array}$ & 5 años & $\mathrm{AU}$ & $\begin{array}{l}\text { Clobetasol, } \\
\text { prednisona, } \\
\text { ciclosporina, } \\
\text { metotrexato, } \\
\text { fototerapia, } \\
\text { simvastatina/ } \\
\text { ezetimiba, } \\
\text { minoxidil, } \\
\text { colecalciferol }\end{array}$ \\
\hline 2 & 14 & M & 5 & No & $\begin{array}{l}\text { Deficiencia de } \\
\text { vitamina D }\end{array}$ & 3 años & $A U$ & $\begin{array}{c}\text { Clobetasol, } \\
\text { prednisona, } \\
\text { colecalciferol, } \\
\text { metotrexato, } \\
\text { simvastatina/ } \\
\text { ezetimiba }\end{array}$ \\
\hline 3 & 16 & M & 3 & No & $\begin{array}{c}\text { Varicela a los } 7 \\
\text { años }\end{array}$ & 3 años & AT & $\begin{array}{c}\text { Prednisona, } \\
\text { betametasona, } \\
\text { nutricosméticos } \\
\text { orales, } \\
\text { latanoprost, } \\
\text { minoxidil }\end{array}$ \\
\hline 4 & 19 & $F$ & 4 & No & Ninguna & 2 años & $\begin{array}{l}\text { Placas } \\
\text { múltiples }\end{array}$ & $\begin{array}{l}\text { Minoxidil, } \\
\text { prednisona, } \\
\text { metotrexato }\end{array}$ \\
\hline
\end{tabular}

AA: alopecia areata; AT: alopecia total; AU: alopecia universal; F: femenino; M: masculino.

Tabla 2. Gravedad de la alopecia según la escala SALT al inicio, a los 3 y 6 meses y efectos adversos

\begin{tabular}{|l|l|l|l|l|l|l|}
\hline Paciente & Efectos adversos & SALT (inicio) & SALT (3 m) & SALT (6 m) & Respuesta al tratamiento & $\begin{array}{l}\text { Satisfacción del } \\
\text { paciente (6 m) }\end{array}$ \\
\hline 1 & Ninguno & $99 \%(\mathrm{~S} 4)$ & $95 \%(\mathrm{~S} 4)$ & $70 \%(\mathrm{~S} 3)$ & Moderada & Insatisfecha \\
\hline 2 & Faringoamigdalitis & $96 \%(\mathrm{~S} 4)$ & $76 \%(\mathrm{~S} 4)$ & $60 \%(\mathrm{~S} 3)$ & Moderada & Satisfecho \\
\hline 3 & Ninguno & $97 \%(\mathrm{~S} 4)$ & $64 \%(\mathrm{~S} 3)$ & $16 \%(\mathrm{~S} 1)$ & Intermedia & Insatisfecho \\
\hline 4 & Ninguno & $70 \%(\mathrm{~S} 3)$ & $25 \%(\mathrm{~S} 2)$ & $19 \%(\mathrm{~S} 1)$ & Intermedia & Satisfecho \\
\hline
\end{tabular}

SALT: Escala de gravedad de alopecia; m: meses.

$99 \%{ }^{12}$; sin embargo, existen pocos estudios en niños y adolescentes. Destaca el trabajo realizado por Craiglow, et al. (2016), que incluyó 13 pacientes con AU y AT de 12 a 17 años de edad, tratados con tofacitinib ( $5 \mathrm{mg}$ vía oral cada $12 \mathrm{~h}$ por un tiempo promedio 6.5 meses), en el que se observó crecimiento de cabello en el $70 \%$, sin efectos adversos graves ${ }^{13}$.

En varios reportes se han descrito casos únicos de jóvenes de 12 a 19 años con AA, tratados con tofacitinib $^{11,14-20}$. La respuesta descrita ha sido completa (tres pacientes), intermedia (dos pacientes) y ausente (un paciente). Estos datos muestran un comportamiento similar a la serie de Craiglow, et al. ${ }^{13}$. En la mayoría de los estudios, el tiempo de duración del tratamiento oscila entre los 5 y los 12 meses, y como principal efecto adverso, las infecciones leves (25.8\%), especialmente de vías aéreas superiores $(16.7 \%)^{12}$, similar a la serie de casos aquí reportada (Tabla 3).

En este trabajo, se observó una respuesta menor al $20 \%$ solo en una paciente. Sin embargo, en este caso se presentaron factores de pobre pronóstico: AU de más de 10 años de evolución (inicio a los 3 años de edad), enfermedad tiroidea autoinmune y falta de respuesta a metotrexato, ciclosporina y fototerapia. 
Tabla 3. Casos reportados de pacientes menores de 20 años con AA tratados con tofacitinib oral y tópico

\begin{tabular}{|c|c|c|c|c|c|c|c|c|}
\hline $\begin{array}{l}\text { País y } \\
\text { añ̃o }\end{array}$ & Autores $^{\text {Ref }}$ & Casos & Edad (años) & Sexo & $\begin{array}{l}\text { Tipo de } \\
\text { alopecia }\end{array}$ & $\begin{array}{c}\text { Duración del } \\
\text { tratamiento (meses) }\end{array}$ & Evolución & $\begin{array}{l}\text { Efectos } \\
\text { adversos }\end{array}$ \\
\hline $\begin{array}{l}\text { EE.UU., } \\
2018\end{array}$ & Patel, et al. ${ }^{14}$ & 1 & 17 & M & $\mathrm{AU}$ & 5 & $\begin{array}{l}\text { Respuesta } \\
\text { intermedia }\end{array}$ & Ninguno \\
\hline $\begin{array}{l}\text { EE.UU., } \\
2018\end{array}$ & Chiang, et al. ${ }^{11}$ & 1 & 17 & M & $\mathrm{AU}$ & 10 & $\begin{array}{l}\text { Respuesta } \\
\text { completa }\end{array}$ & $\begin{array}{l}\text { Infección } \\
\text { por herpes } \\
\text { zóster }\end{array}$ \\
\hline $\begin{array}{l}\text { EE.UU., } \\
2018\end{array}$ & $\begin{array}{l}\text { Putterman y } \\
\text { Castelo }^{15}\end{array}$ & 11 & $4-16$ & F/M & $\begin{array}{l}\mathrm{AA}, \mathrm{AT}, \\
\mathrm{AU}\end{array}$ & 8 & $\begin{array}{l}\text { Respuesta } \\
\text { intermedia }\end{array}$ & $\begin{array}{l}\text { Sin efectos } \\
\text { adversos }\end{array}$ \\
\hline $\begin{array}{l}\text { EE.UU., } \\
2017\end{array}$ & McKesey, et al. ${ }^{16}$ & 1 & 19 & $\mathrm{~F}$ & $\mathrm{AU}$ & 12 & $\begin{array}{l}\text { Respuesta } \\
\text { completa }\end{array}$ & Ninguno \\
\hline $\begin{array}{l}\text { Alemania, } \\
2017\end{array}$ & Mrowietz, et al. ${ }^{17}$ & 1 & 20 & $\mathrm{~F}$ & $A U$ & 9 & $\begin{array}{l}\text { Respuesta } \\
\text { completa }\end{array}$ & $\begin{array}{l}\text { Infección } \\
\text { por herpes } \\
\text { zóster y } \\
\text { constipación }\end{array}$ \\
\hline $\begin{array}{l}\text { EE.UU., } \\
2017\end{array}$ & Ibrahim, et al. ${ }^{18}$ & 2 & 20 & $\mathrm{~F} / \mathrm{M}$ & $\mathrm{AU}$ & $4-7$ & Sin respuesta & Ninguno \\
\hline $\begin{array}{l}\text { EE.UU., } \\
2017\end{array}$ & Castelo-Soccio ${ }^{19}$ & 8 & 8-19 & F/M & $A U$ & $5-18$ & $\begin{array}{l}\text { Respuesta } \\
\text { intermedia }\end{array}$ & Ninguno \\
\hline $\begin{array}{l}\text { EE.UU., } \\
2017\end{array}$ & Craiglow, et al. ${ }^{13}$ & 13 & $12-17$ & $\mathrm{~F} / \mathrm{M}$ & $\begin{array}{l}\text { AA, AT, } \\
\text { AU }\end{array}$ & $2-16$ & $\begin{array}{l}\text { Nueve } \\
\text { respondedores } \\
\text { Cuatro no } \\
\text { respondedores }\end{array}$ & Ninguno \\
\hline $\begin{array}{l}\text { Brasil, } \\
2016\end{array}$ & $\begin{array}{l}\text { Scheinberg y } \\
\text { Ferrera }^{20}\end{array}$ & 2 & 20 & $\mathrm{~F}$ & $\mathrm{AU}$ & 9 & $\begin{array}{l}\text { Respuesta } \\
\text { intermedia }\end{array}$ & $\begin{array}{l}\text { No } \\
\text { reportados }\end{array}$ \\
\hline
\end{tabular}

AA: alopecia areata; AT: alopecia total; AU: alopecia universal; F: femenino; M: masculino.

Con respecto al uso tópico, Putterman y Castelo-Soccio reportaron 11 pacientes con AA (AT y AU) de 4 a 16 años, tratados con tofacinib en solución liposomal. Estos autores observaron una mejoría de moderada a intermedia, con tasas de respuesta mayores al $50 \%$, sin efectos secundarios sistémicos y un caso de dermatitis por contacto $^{15}$.

No hay estudios que reporten los efectos secundarios del tofacitinib a largo plazo. De acuerdo con Castañeda, et al., en un estudio donde se evaluó la seguridad de este fármaco en pacientes latinoamericanos con artritis reumatoide, se observaron, como principales efectos adversos, tuberculosis, infecciones oportunistas, herpes zóster y malignidad (cáncer de piel no melanoma, linfo$\mathrm{ma})^{21}$. En los estudios de laboratorio, se han reportado anormalidades en la cuenta linfocitaria, de neutrófilos, anemia, elevación del colesterol y transaminasemia ${ }^{22}$.

Considerando que las recurrencias al suspender el tratamiento llegan al $100 \%$, se debe considerar el tratamiento continuo. Sin embargo, considerando los efectos descritos a largo plazo, algunos autores sugieren su uso por periodos cortos y en forma intermitente, para conservar la posibilidad del crecimiento del cabello y brindarles a los pacientes la oportunidad de recuperación ${ }^{12,13,23}$.

Es importante resaltar que a todos los pacientes candidatos al tratamiento con tofacitinib se les deben realizar estudios de escrutinio: biometría hemática completa, pruebas de función hepática, química sanguínea (incluyendo un perfil lipídico, panel viral para virus de la hepatitis, $B, C$ y VIH), PPD e IFN- $\gamma$ (debido a que México es una zona con alta prevalencia de infección por Mycobacterium tuberculosis). Es recomendable que los pacientes susceptibles a infección por herpes zóster, como los adultos mayores, sean vacunados. Durante el seguimiento, es necesario un control periódico de estudios de laboratorio.

Se debe interrogar a los pacientes en cada consulta y vigilar, durante el seguimiento, cualquier dato de infección o malignidad, realizar la exploración dermatológica completa en busca de cáncer de piel y evitar, en la medida de lo posible, el uso concomitante de antiinflamatorios no esteroideos, corticoides o metotrexato, debido al riesgo de erosiones de la mucosa gástrica e intestinal. 
Como conclusión, y con base en estos resultados, se considera que el tofacitinib es una alternativa para el manejo de AA grave. Por otro lado, es importante considerar la relación costo/beneficio e individualizar cada caso. Son necesarios más estudios para determinar el efecto terapéutico máximo que el tratamiento con tofacitinib puede lograr, las posibilidades de recurrencia de la enfermedad al suspender el medicamento y los efectos adversos y a largo plazo.

\section{Responsabilidades éticas}

Protección de personas y animales. Los autores declaran que para esta investigación no se han realizado experimentos en seres humanos ni en animales.

Confidencialidad de los datos. Los autores declaran que han seguido los protocolos de su centro de trabajo sobre la publicación de datos de pacientes.

Derecho a la privacidad y consentimiento informado. Los autores han obtenido el consentimiento informado de los pacientes o individuos referidos en el artículo. Este documento obra en poder del autor de correspondencia.

\section{Conflicto de intereses}

Los autores declaran no tener ningún conflicto de intereses.

\section{Financiamiento}

Todo el material utilizado se encuentra dentro del cuadro básico y recursos de la Secretaría de la Defensa Nacional.

\section{Bibliografía}

1. Strazzulla LC, Wang E, Avila L, Lo Sicco K, Brinster N, Crristiano A, et al Alopecia areata: disease characteristics, clinical evaluation and new perspectives on pathogenesis. J Am Acad Dermatol. 2018;78:1-12.
2. Gip L, Lodin A, Molin L. Alopecia areata. A follow-up investigation of outpatient material. Acta Derm Venereol (Stockh). 1969;49:180-8.

3. Jang YH, Hong NS, Moon SY, Eun DH, Lee WK, Chi SG, et al. Long-term prognosis of alopecia totalis, universalis. A longitudinal study with more than 10 years of follow-up: better than reported. Dermatology. 2017; 233:250-6.

4. Tosti A, Bellavista S, lorizzo M. Alopecia areata: a long term follow-up study of 191 patients. J Am Acad Dermatol. 2006;55:438-41.

5. Messenger AG, McKillop J, Farrant P, McDonagh AJ, Sladden M. British Association of Dermatologists' guidelines for the management of alopecia areata 2012. Br J Dermatol. 2012;166:916-26.

6. Gupta AK, Carviel J, Abramovits W. Treating alopecia areata: current practices versus new directions. Am J Clin Dermatol. 2016;18:67-75.

7. Madani S, Shapiro J. Alopecia areata update. J Am Acad Dermatol 2000;42:549-66

8. Xing L, Dai Z, Jabbari A, Cerise J, Higgins C, Gong W, et al. Alopecia areata is driven by cytotoxic T lymphocytes and is reversed by JAK inhibition. Nat Med. 2014;20:1043-9.

9. Wesh K, Holstein J, Laurence A, Ghoreschi K. Targeting Jak/STAT signalling in inflammatory skin diseases with small molecule inhibitors. Eur J Immunol. 2017;47:1096-107.

10. Craiglow BG, King BA. Killing two birds with one stone: oral tofacitinib reverses alopecia universalis in a patient with plaque psoriasis. 2014; 134:2988-90.

11. Chiang A, Ortenzio F, Juhasz ML, Yu V, Mesinkovska NA. Balance of tofacitinib efficacy and disease flare in the treatment of alopecia universalis: a case report and review of the literature. JAAD Case Rep. 2018;4:733-6.

12. Liu LY, Craiglow BG, Dai F, King BA. Tofacitinib for the treatment of severe alopecia areata and variants: a study of 90 patients. J Am Acad Dermatol. 2017;76:22-8.

13. Craiglow BG, Liu LY, King BA. Tofacitinib for treatment of alopecia areata and variants in adolescents. J Am Acad Dermatol. 2017:76:29-32.

14. Patel NU, Oussedik E, Grammenos A, Pichardo-Geisinger R. A case report highlighting the effective treatment of alopecia universalis with tofacitinib in an adolescent and adult patient. J Cutan Med Surg. 2018;22:439-42.

15. Putterman E, Castelo-Soccio L. Topical $2 \%$ tofacitinib for children with alopecia areata, alopecia totalis, and alopecia universalis. J Am Acad Dermatol. 2018;78:1207-9.

16. McKesey J, Blankenship DW, Jamison M, DeWitt C. Alopecia universalis successfully treated with tofacitinib. J Am Acad Dermatol. 2017;76:AB38.

17. Mrowietz U, Gerdes S, Gläser R, Schröder O. Successful treatment of refractory alopecia areata universalis and psoriatic arthritis, but not of plaque psoriasis with tofacitinib in a young woman. Acta Derm Venereol. 2017:97:283-4.

18. Ibrahim O, Bayart CB, Hogan S, Piliang M, Bergfeld WF. Treatment of alopecia areata with tofacitinib. JAMA Dermatol. 2017;153:600-2.

19. Castelo-Soccio LD. Experience with oral tofacitinib in 8 adolescent patients with alopecia universalis. J Am Acad Dermatol. 2017;76:754-5.

20. Scheinberg M, Ferreira SB. Reversal of alopecia universalis by tofacitinib: a case report. Ann Intern Med. 2016:165:750-1.

21. Castañeda O, Romero FJ, Salinas A, Citera G, Mysler E, Rillo O. Safety of tofacitinib in the treatment of rheumatoid arthritis in Latin America compared with the rest of the world population. J Clin Rheumatol. 2017; 23:193-9.

22. US Food and Drug Administration Advisory Committee Meeting Tofacitinib for the treatment of rheumatoid arthritis: NDA 203214 briefing document; 2018. Disponible en: https://www.fda.gov/media/111372/download.

23. Kennedy Crispin M, Ko JM, Craiglow BG, Li S, Shankar G, Urban JR, et al. Safety and efficacy of the JAK inhibitor tofacitinib citrate in patients with alopecia areata. JCI Insight. 2016;1:e89776. 